\title{
Repair After Tears of the Retinal Pigment Epithelium
}

\author{
E. L. CHUANG* and A. C. BIRD \\ London
}

\begin{abstract}
Summary
Sixty-three eyes (in 59 patients) with tears of detached retinal pigment epithelium have been studied for a period of up to 10 years after the acute event. In the majority of cases a plaque of fibrous tissue was laid down gradually in the bed of the tear. In a few eyes the inner surface of Bruch's membrane appeared to remain relatively unaltered for up to 2 years, and in four eyes it was recovered with pigmented tissue resembling normal retinal pigment epithelium. These and other changes progressively obscured the distinctive feature of the original lesion; the nature of the original lesion was evident at 6 months in all cases, by 1 year it was recognisable in 94 per cent, by 2 years in 77 per cent and by 6 years in none. Choroidal neovascularisation was detected or suspected in one third of cases in the acute stages and developed in others weeks to months after the acute event. Alteration of the morphology occurred more quickly, and there was greater scarring, in those with new vessels or significant blood in the subretinal space, when compared with those without such features. Visual acuity was surprisingly good in many patients immediately after the tear. However, loss of vision accompanied the reactive tissue remodelling, and no case had long term retention of good visual acuity.
\end{abstract}

Since first described in $1981,{ }^{1}$ rips or tears at the edge of retinal pigment epithelial detachments have been recognised increasingly as a cause of severe loss of central vision in agerelated macular degeneration. Subsequent publications have redescribed the fundus and angiographic features characteristic of an acute tear occurring either spontaneously, or following laser photocoagulation. ${ }^{2-14}$ The long term evolution of the lesion has yet to be fully described, although the long term visual prognosis is reported to be poor. ${ }^{9}$

The pathogenesis of such lesions is not well understood. It has been suggested that the nature of the pigment epithelial detachment may predispose to the tear. ${ }^{1}$ Gass has reported that sub-pigment epithelial new vessels are common in such lesions; ${ }^{6}$ he implied that con- traction of the fibrovascular tissue on the outer surface of the detached tissues may induce tension sufficient to cause a rupture of the pigment epithelial layer.

This retrospective study was undertaken to document the long term evolution of such lesions. The prevalence of new vessels was sought specifically at different stages after the tear to assess the potential importance of neovascularisation to the pathogenesis of the lesion.

\section{Study Patients}

Sixty-three eyes of 59 patients were identified with clear evidence of a pigment epithelial tear, and with reliable data upon which the chronological age of the lesion could be judged. In 1986, all subjects were invited for

Correspondence to: Department of Clinical Ophthalmology, Institute of Ophthalmology, Moorfields Eye Hospital, City Road, London EC1V 2PD.

*Current address: Bascom Palmer Eye Institute, Miami, Florida 33101.

This study was supported in part by Medical Research Council grant number G.8320019 N. 
further examination in order to provide as long a period of review as possible. The mean total period of review from the time of the tear for these eyes was 25.5 months, with a range of 1 to 126 months. There were 33 females aged 56 to 84 years (mean 70.6 years), and 26 males aged 55 to 82 years (mean 71 years).

\section{Methods}

For the purpose of accurately correlating characteristics of the lesion with the age of the tear, details from clinical examination, patients records, stereo colour photography and fluorescein angiography were analysed.

The time at which the tear occurred, hereafter designated as the "event", was estimated on the basis of symptoms, clinical records and examination findings. When present, a subjective history of an acute visual disturbance was accepted as indicating the time of an acute rip. For other eyes, consecutive examinations separated by no more than 4 weeks were used to establish the time of the event, designated as the midpoint of the interval during which a tear occurred.

Initial analysis showed no significant modification in the appearance of tears within the first 6 weeks following the event. This period was designated the "acute" phase; there were 27 eyes of 26 patients which were seen during this interval. In 36 eyes of 33 patients, the first examination following the rip was carried out more than 6 weeks after the event. These together with 18 eyes in the acute phase were studied to identify the repair processes.

Choroidal neovascularisation was specifically sought, and was considered to be definitely present when angiography demonstrated a subretinal neovascular complex. It was suspected in the presence of subretinal or intraretinal exudation, or if the pre-rip pigment epithelial detachment had characteristics thought to imply the presence of subretinal new vessels (such as radial choroidal folds, notching, subpigment epithelial blood or uneven subretinal fluorescence).

\section{Results}

\section{Acute Phase}

Subretinal blood was present on the initial examination in 10 of 27 eyes with acute tears; in 5 of these, the bed of the rip was completely covered by blood, which gradually resorbed revealing features typical of a pigment epithelial tear (Fig. 1). No eyes subsequently developed more than flecks of new subretinal blood in the acute phase.

A distinctive orange appearance to the bed of the tear was present in 17 of the 22 eyes without an obscuring layer of blood (Fig. 2). The bed of the tear was evenly and intensely hyperfluorescent in 17 of 18 eyes in which transit fluorescein angiograms were recorded in the affected eye. The peripheral torn edge of the pigment epithelium presented a sharp, scalloped border which was clearly evident on fundus examination, colour fundus photographs and/or angiography in all but 5 of 22 eyes in which blood did not cover the entire bed (Fig. 3).

In many but not all acute eyes, there was persistent elevation of the central pigment epithelium with folds oriented in a parallel fashion to the torn edge of the tissue. Subretinal fluid was commonly present over the bed of the tear and adjacent pigment epithelium in the earliest period following a rip. This fluid tended to be dependent but did not always stain in the late phases of fluorescein angiography.

In 15 eyes of the acutely observed group the pigment epithelial detachment was documented prior to tearing. Of these, four had definite choroidal new vessels within the detachment before the tear and one developed such evidence by the time of the first post-rip examination. Thus, there was evidence of choroidal neovascularisation complicating a pigment epithelial detachment prior to the rip in approximately one-third of eyes. Of 12 eyes not seen pre-rip, but first examined within 6 weeks of the event, five contained angiographic evidence of new vessels on this examination ( 3 definite and 2 suspect).

No eyes developed fibrous tissue in the bed of the tear or hyperpigmentation secondary to pigmentary hyperplasia during the acute phase.

\section{Repair Phase}

Resorption of subretinal blood deposited at the time of the tear continued in the absence of new haemorrhage. Occasionally new blood 

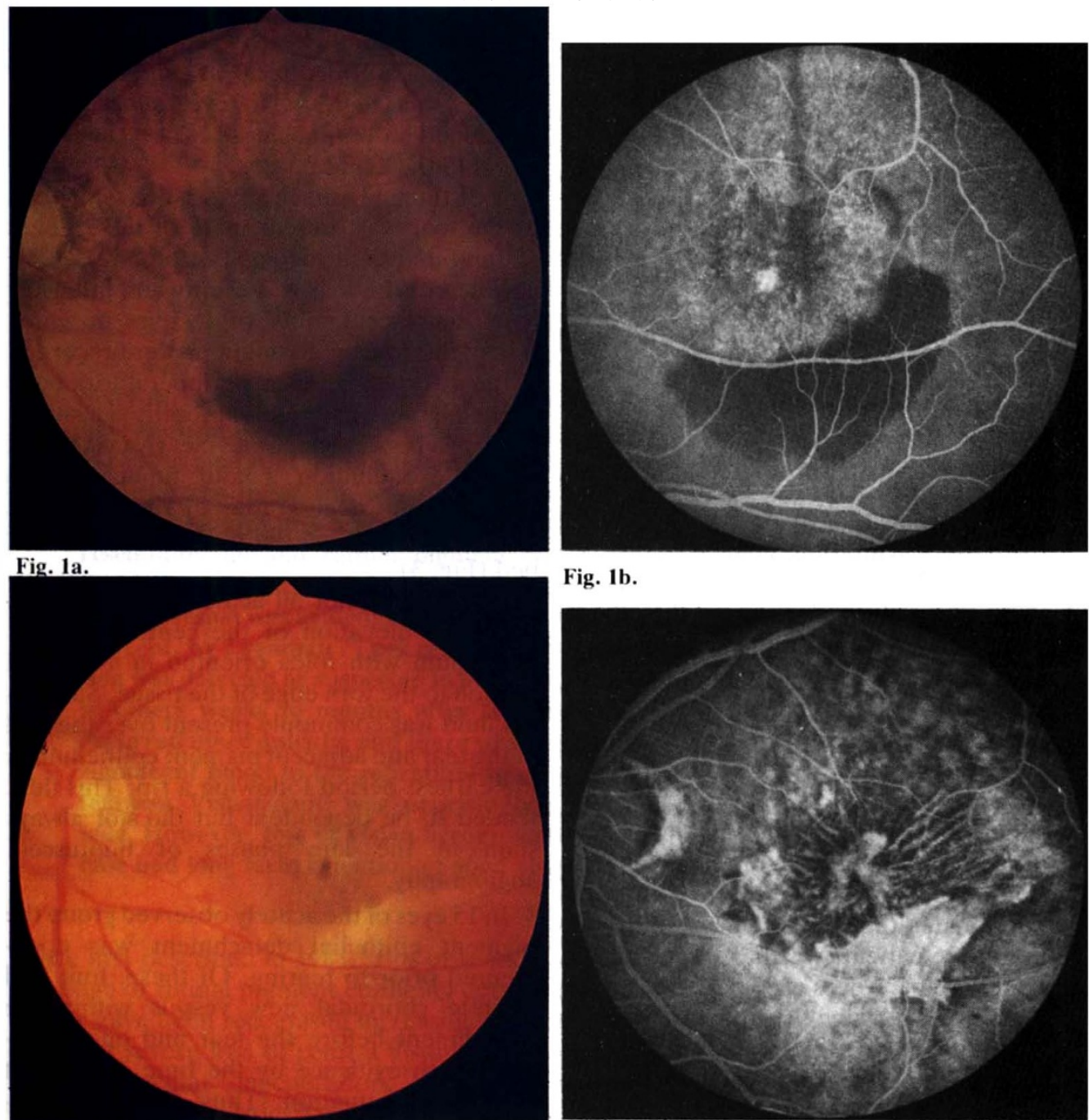

Fig. 1 .

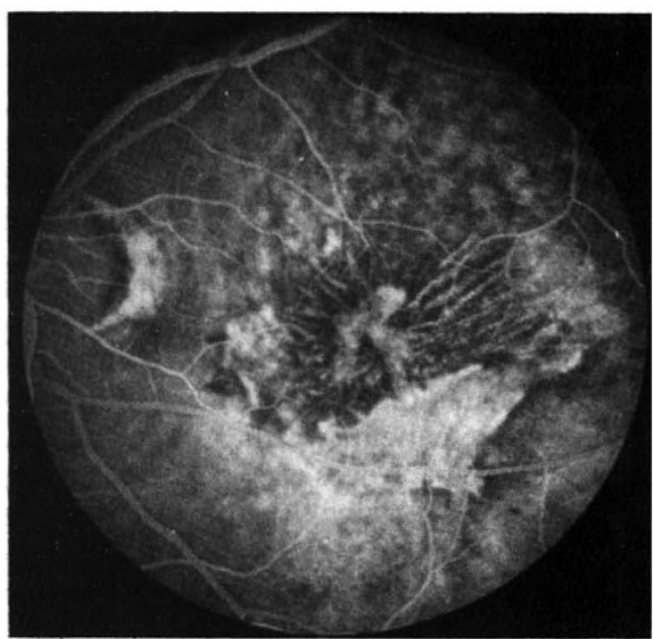

Fig. 1c.

Fig. $1 d$.

Fig. 1. Photograph and fluorescein angiogram 5 weeks after visual loss, showing blood covering the bed of a tear at the lower edge of a pigment epithelial detachment $(l a, b)$. Seven months later resorption of the haemorrhage allowed a view of the inner surface of Bruch's membrane which had become covered with a plaque of fibrous tissue $(l c, d)$.

appeared at the margins of the lesion due to apparent extension of the tear in a few eyes, but rarely due to choroidal neovascularisation.

In 7 of the 22 lesions without significant subretinal blood, the subretinal fluid absorbed by the end of the acute phase, and an additional four had flattened by 8 weeks. After the initial 6 weeks, the orange appearance of the bed was gradually altered. Of 29 eyes examined 8 weeks after the event only 5
(18 per cent) demonstrated this characteristic, although a few retained the orange appearance of the bed for up to 14 months. Loss of this feature occurred as the bed of the tear was replaced by pigmented or scar tissue. All or part of the denuded Bruch's membrane was observed to become covered by tissue resembling normal retinal pigment epithelium in 4 eyes. In 2 there was a radial pattern of hypoand hyperfluorescence (Fig. 4). In one eye examined initially 2 weeks after a pigment 


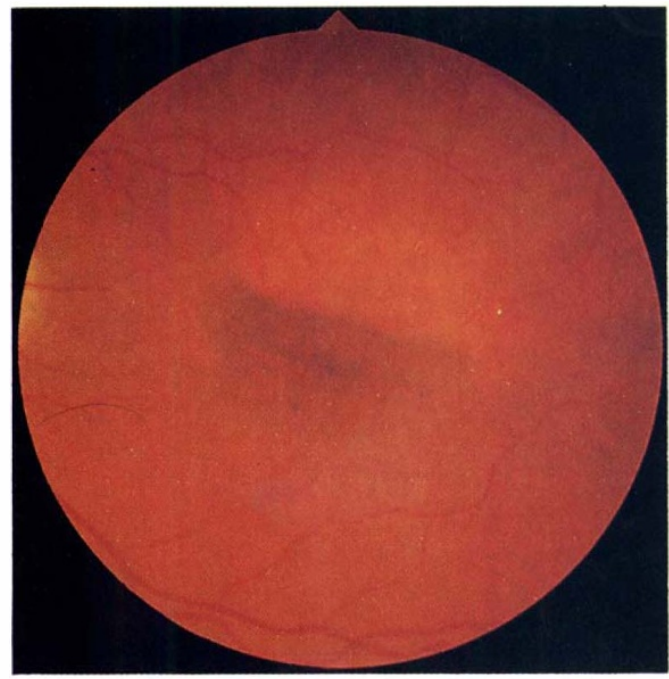

Fig. 2a.

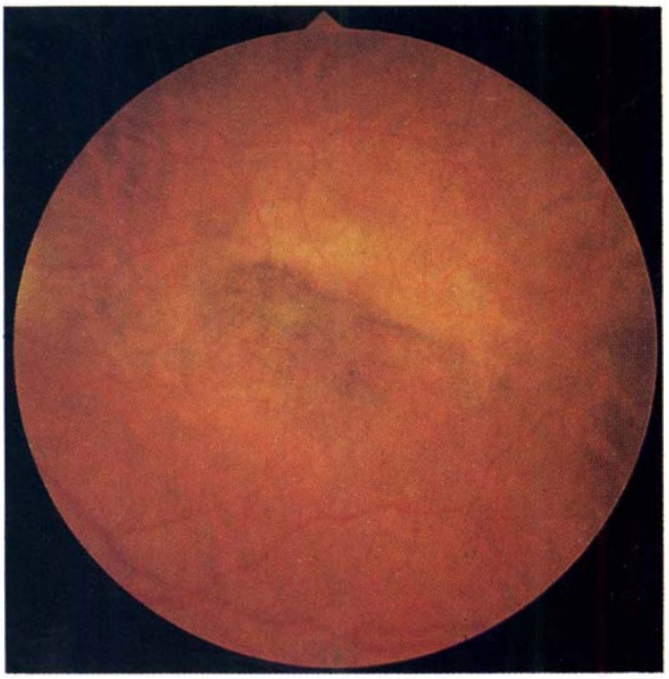

Fig. 2 b.

Fig. 2. Photographs showing the orange appearance of the bed of an acute tear and deposition of fibrous tissue on the inner surface of the bend of the tear over a period of 2 years $(2 a, b)$.

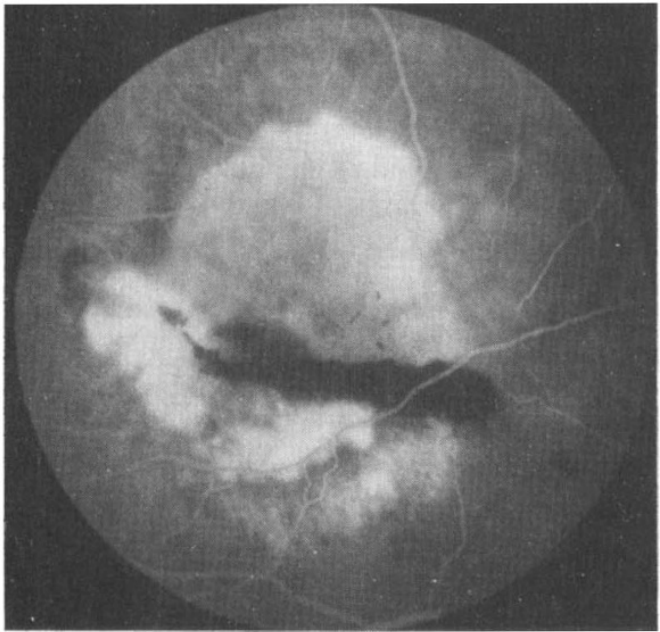

Fig. 3a.

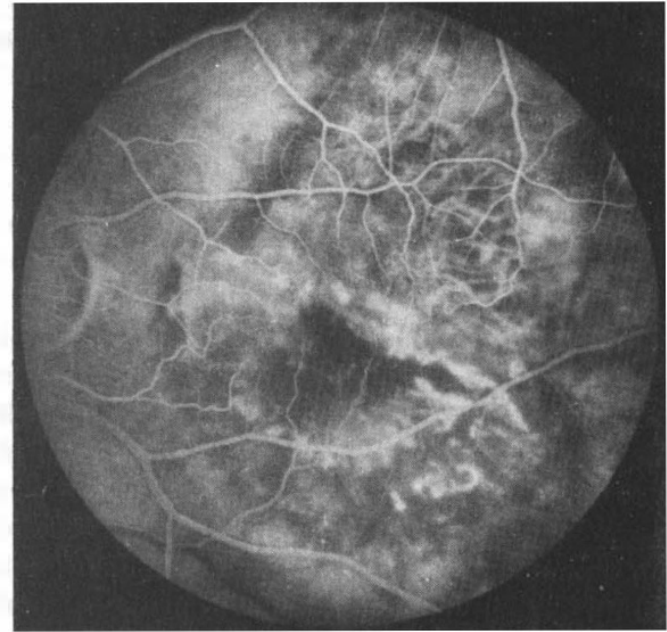

Fig. 3b.

Fig. 3. Even, intense hyperfluorescence in the bed of a subacute tear 4 months after the acute event also demonstrating the scalloped edge of an RPE tear (3a). Eight months later, atrophy of the choriocapillaris reveals large choroidal vessels $(3 \mathrm{~b})$.

epithelial tear (precipitated by laser photocoagulation), the peripheral border of the torn pigment epithelium was noted to move posteriorly towards its original location (Fig. 5).

In the majority of cases the bed of the tear was replaced by flat, yellow material resembling a thin layer of fibrous tissue which matched precisely the area of bare Bruch's membrane (Fig. 2). In the absence of aggres- sive new vessels or massive subretinal blood this response was confined to the bed of the rip, and left the residual mound of pigment epithelium surrounded by fibrous tissue (Fig. 6). The basic configuration of the rip bed and adjacent tissue was thus preserved for many months. Thereafter progressive development of dense fibrous tissue camouflaged the surface and precise borders of the previous bed. 


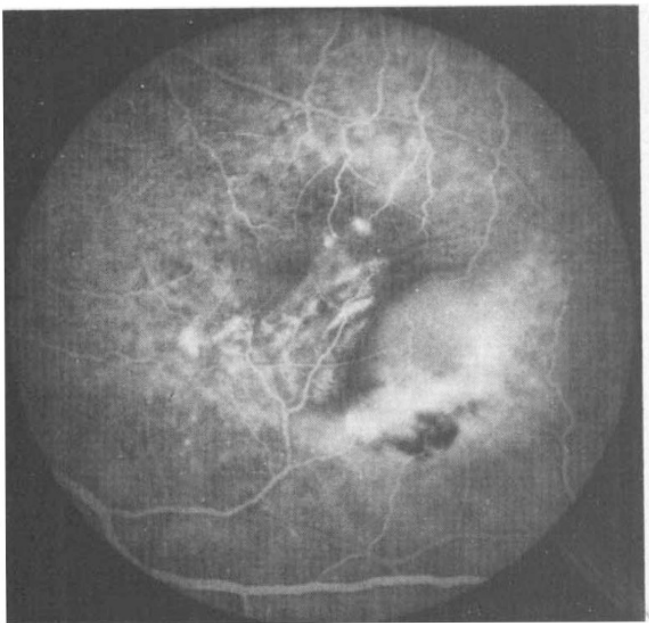

Fig. 4a.

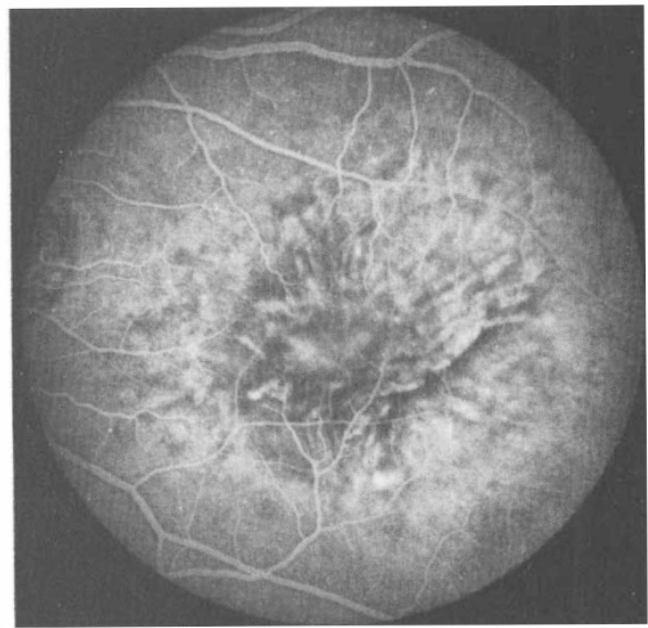

Fig. 4 b.

Fig. 4. Fluorescein angiogram 1 day after a rip at the inferior temporal edge of a pigment epithelial detachment showing intense hyperfluorescence corresponding with the bed of the rip (4a). Within 9 months the inner surface of Bruch's membrane appears to have been re-covered by retinal pigment epithelium (arrows) which shows linear hyperfluorescence along the line of presumed slide $(4 \mathrm{~b})$. The linear fluorescence of the detached retinal pigment epithelium may be due to folds in the redundant tissue and/or to sub-retinal pigment epithelial neovascularisation.

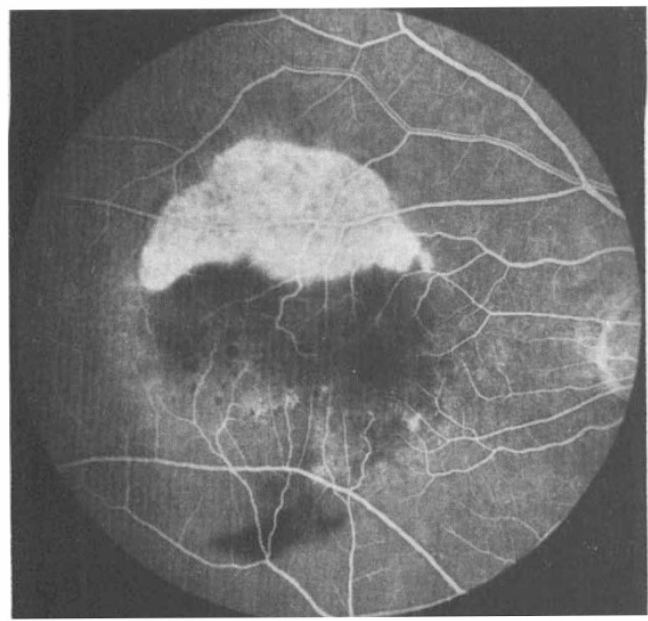

Fig. 5a.

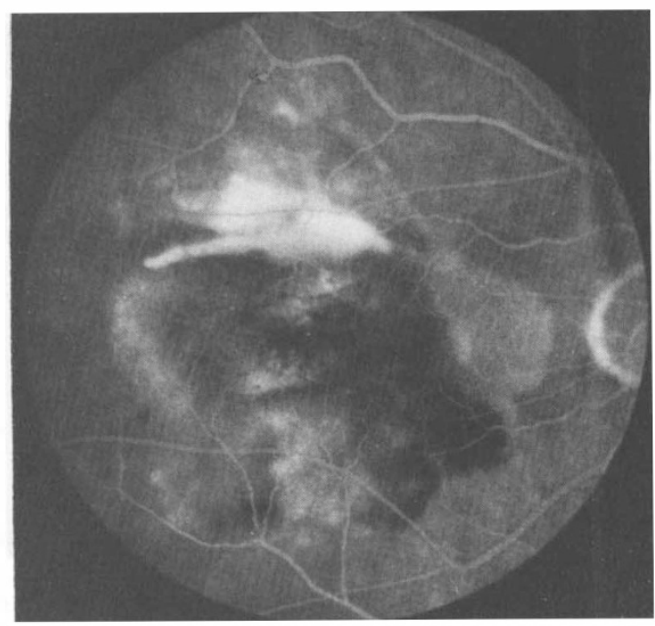

Fig. 5 b.

Fig. 5. Fluorescein angiogram 2 weeks after loss of visual acuity showing hyperfluorescence at the level of Bruch's membrane following a tear at the superior border of a pigment epithelial detachment (5a). One month later the upper part of the bed of the tear was re-covered with relatively normal appearing pigment epithelium (5b).

Hyperpigmentation within the scar, primarily of the residual central pigment epithelial mound, did not appear in the first year. Only one eye demonstrated such pigment 2 years after the event, but virtually all eyes examined 3 years after the event did so.

In tears complicated by significant bleeding or aggressive neovascularisation, the heavily fibrotic appearance tended to appear earlier causing faster disappearance of the pathognomonic signs than in those lesions lacking these features.

The fluorescein angiographic appearance reflected the tissue changes. Abnormal perfusion of the choriocapillaris, suggesting slow flow or closure, was identified in all eyes in 


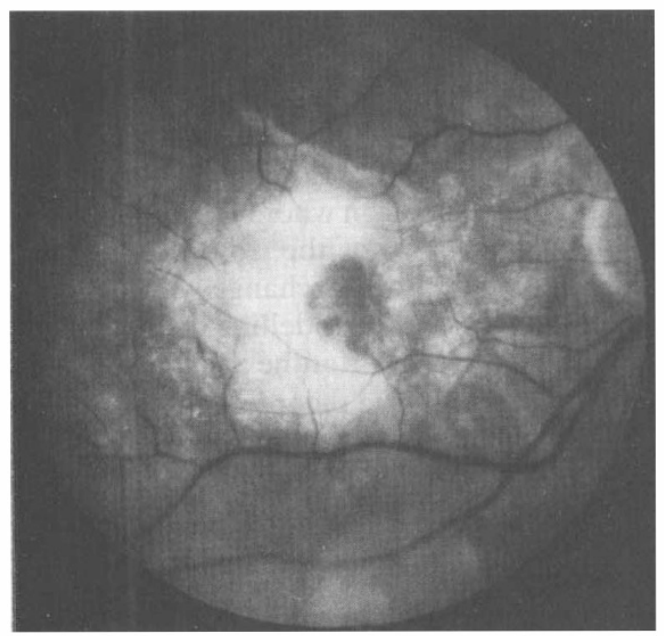

Fig. 6. Red free photograph of a pigment epithelial tear 3 years after the acute event. The bed of the tear and the residual pigment epithelium are still well defined and the nature of the original lesion evident.

which the bed of the tear was visible (Fig. 3). In those cases in which fibrous tissue replaced the defect, there was even and slow appearance of hyperfluorescence corresponding with the scar.

Choroidal new vessels were found in 16 eyes and suspected in three of 36 eyes first examined after the first 6 weeks. Two eyes developed such evidence anew since presentation in the acute phase. Thus 61 per cent of all eyes showed evidence of new vessel formation at some stage in the period of review.

After 1 year, 94 per cent, and after 2 years, 77 per cent of eyes retained features characteristic enough to imply a rip as the origin of the lesion. None of the lesions seen 6 years or more after the event could reliably be recognised as having originated as a pigment epithelial tear.

\section{Vision}

The visual acuity in patients with pigment epithelial tears varied widely. During the acute period, vision was often good (6/9 to $6 / 18)$ in eyes in which there was pigment epithelium beneath the fovea. Loss of acuity at the time of the rip was limited to one line or was unchanged in 11 of 28 eyes ( 23 patients) in whom visual function had been documented before and after the rip. Surprisingly, an improvement in acuity was recorded in four of these eyes on the first visit after the event. Of patients seen within 6 months of a rip, and who had previously lost vision in the contralateral eye, about 50 per cent did not report or recall a sudden visual disturbance.

Progressively fewer patients had good visual acuity as the period of review increased: only nine of 38 examined at one year and three of 26 examined at 2 years had an acuity of better than $6 / 60$. Visual acuity was worse than $6 / 60$ in all affected eyes more than two years following the event.

\section{Discussion}

To date 127 eyes with tears of the retinal pigment epithelium have been described in the literature $;^{1-14}$ these reports have been related largely to the acute lesion. Among these are single case reports of tears in association with retinal detachment with proliferative vitreoretinopathy and a chorioretinal scar, but the setting of most importance is that complicating detachment of the retinal pigment epithelium within the spectrum of age-related macular degeneration. Over a period of review of at least one year, 10 per cent of eyes with pigment epithelial detachments suffered tears. ${ }^{9}$

Although sequential changes following this complication of RPE detachment have been documented, no precise data exist concerning the time sequence or frequency of these modifications. Our observations show that there is considerable tissue remodelling in the lesion over several years. Many eyes with acute tears developed a late appearance indistinguishable from that of primary neovascular disciform lesions as cicatrisation gradually concealed the true nature of the original lesion. However, the fundus and angiographic features are virtually pathognomonic within the first 6 months in all lesions not obscured by blood, and in the majority of eyes for up to 2 years.

The nature of the initial repair process varied. In most cases the pigment epithelial defect was replaced by a plaque of fibrous tissue. In a few lesions the inner surface of Bruch's membrane became re-covered by tissue resembling normal retinal pigment epithelium. These areas were hypopigmented, and two had poorly defined, radially oriented lines of hypo- and hyperfluorescence on fluor- 
escein angiography. In a small number of patients, the appearance did not alter for some months after the initial rip, suggesting that Bruch's membrane remained denuded or became re-epithelialised by non-pigmented cells.

The pattern of the healing process has been compared with that reported after experimental trauma to the pigment epithelium. ${ }^{1}$ In small lesions the defect is covered by sliding over the area with limited division of pigment epithelial cells. ${ }^{15}$ This would account for the apparent re-epithelialisation of Bruch's membrane, and the radial lines observed in 2 eyes may be a product of sliding. In larger defects the pigment epithelial cells proliferate and become fibroblastic. ${ }^{16}$ Such a process would cause the bed of a rip to be replaced by fibrous tissue as was seen in the majority of cases. Accumulation of fibrous tissue and hyperpigmentation occurred for several years after the acute event indicating prolonged proliferative activity.

There is also the possible contribution of blood-derived fibroblasts, particularly when choroidal new vessels and/or blood itself is present. In lesions with rapidly growing neovascular tissue there was early modification of the appearance with erosion of the limits of the bed of the tear, and loss of a recognisable central flap of pigment epithelium. Thus the analogy between experimental pigment epithelial trauma and tears is less evident in the presence of neovascularisation.

The choroidal new blood vessels were prominent in the early stages of repair in only one third of cases, and many lesions remained apparently avascular throughout their evolution. Neovascularisation became detectable in some lesions some time after the acute event so that a majority of patients had new vessels at some stage during the evolution of the disorder. We acknowledge that the absence of detectable new vessels does not exclude their presence. Nevertheless, the failure to demonstrate new vessels in a large proportion of lesions casts doubt on choroidal neovascularisation as the primary pathogenetic mechanism in the genesis of retinal pigment epithelial tears. This is not to deny that blood vessels may play a pathogenetic role in some cases, and it is evident that in many, neovascularisation had a major influence on the evolution of the lesion.

Vision was surprisingly good soon after the event, and in over one third of acute cases seen visual acuity was $6 / 18$ or better. This was observed in patients in whom the tear was not sub-foveal such that the foveal receptors maintained metabolic exchange with pigment epithelium. Tissue remodelling caused acuity to be lost often long after the acute event such that in no case did we record an acuity of $6 / 60$ or better beyond 2 years. The poor long term visual prognosis reflects the prolonged phase of cicatrisation in this disorder.

\section{References}

${ }^{1}$ Hoskin A, Bird AC, Sehmi K: Tears of detached retinal pigment epithelium. $\mathrm{Br} J$ Ophthalmol 1981, 65: 417-22.

${ }^{2}$ Coscas G, Quentel G, Pinon F, Soubrane G: Déchirure spontanée de l'épithelium pigmentaire dans la région maculaire. Bull Soc Ophthalmol Fr 1982, 82: 815-20.

${ }^{3}$ Cantrill HL, Ramsay RC, Knobloch WH: Rips in the pigment epithelium. Arch Ophthalmol 1983, 101: 1074-9.

${ }^{4}$ Green SN and Yarian D: Acute tear of the retinal pigment epithelium. Retina 1983, 3: 16-20.

${ }^{5}$ Decker WL, Sanborn GE, Ridley M, Annesley WH, Sorr EM: Retinal pigment epithelial tears. Ophthalmology 1983, 90: 507-12.

${ }^{6}$ Gass JDM: Pathogenesis of tears of the retinal pigment epithelium. Br J Ophthalmol 1984, 68: 513-9.

${ }^{7}$ Gass JDM: Retinal pigment epithelial rip during krypton red laser photocoagulation. $A m \mathrm{~J}$ Ophthalmol 1984, 98: 700-6.

${ }^{8}$ Swanson DE, Kalina RE, Guzak SV: Tears of the retinal pigment epithelium. Retina 1984, 4: 11518.

${ }^{9}$ Casswell AG, Kohen D, Bird AC: Retinal pigment epithelial detachments in the elderly: classification and outcome. Br J Ophthalmol 1985, 69: 397-403.

${ }^{10}$ Sunakawa $M$ and Tsukahara I: Tear of the retinal pigment epithelium and serous retinal detachment. Am J Ophthalmol 1985, 100: 488-9.

11 Krishan NR, Chandra SR, Stevens TS: Diagnosis and pathogenesis of retinal pigment epithelial tears. Am J Ophthalmol 1985, 100: 698-707.

${ }^{12}$ De Laey JJ and Riems D: Ripping of detached retinal pigment epithelium in senile macular degeneration. Bull Soc Belge Ophthalmol 1985, 207: 27-35.

13 Tutein Nolthenius PA and Deutman AF: Rips of the retinal pigment epithelium. Int Ophthalmol 1985, 8: $19-23$. 
${ }^{14}$ Traboulsi EI and Jalkh AE: Retinal pigment epithelium tear as a cause of vitreous haemorrhage. Ann Ophthalmol 1985, 17: 228-37.

${ }^{15}$ Marshall J and Mellerio J: Disappearance of the retroepithelial scar tissue from ruby laser photocoagulation. Exp Eye Res 1971, 93: 173-4.

${ }^{16}$ Marshall $\mathbf{J}$ and Mellerio J: Laser irradiation of retinal tissue. Br Med Bull 1970, 26: 156-60. 